\title{
Manejo anestésico en la enfermedad de Von Recklinghausen. Reporte de un caso
}

\author{
MIGUEL CALVA MALDONADO', SILVIA ZEPEDA OLIVERA², JULIO CESAR PABLO YÁÑEZ³, \\ FABIAN FRAGOSO AVILÉS4
}

\begin{abstract}
Von Recklinghausen syndrome: Case report and anesthesis management

Von Recklinghausen disease or neurofibromatosis type I is a disease characterized by the tendency to form tissue tumors of the ectoderm and mesoderm. There are several anesthetic implications that include altered sensitivity to succinylcholine and non-depolarizing neuromuscular relaxants, in addition to alterations in the normal anatomy of the airway; we present the case of a 58 year old patient scheduled for the resection of the right temporo-parietal cutaneous tumor. Succinylcholine was used as a muscle relaxant. Despite its short half-life, no subsequent doses were required during the treatment period duration of surgery; Therefore, an altered sensitivity towards muscle relaxants of depolarizing type was corroborated in this type of patients.
\end{abstract}

\section{RESUMEN}

La enfermedad de Von Recklinghausen o neurofibromatosis tipo I es una enfermedad caracterizada por tendencia a la formación de tumores de tejidos del ectodermo y mesodermo. Existen diversas implicaciones anestésicas que incluyen la sensibilidad alterada a la succinilcolina y a relajantes neuromusculares nodespolarizantes, además de alteraciones en la anatomía normal de la vía aérea;

\section{Key words:}

Von Recklinghausen, neurofibromatosis, succinylcholine.

\section{Palabras clave:}

Von Recklinghausen, neurofibromatosis, succinilcolina.

\footnotetext{
Autor, Profesor investigador tipo C asociado BUAP profesor adjunto del curso de anestesiología.

2 Coautor, Profesor investigador tipo C asociado BUAP profesor titular del curso de anestesiología.

3-4 Colaborador Profesor Tutor del curso de anestesiología.
}

Conflicto de Interés: No se presenta ningún conflicto de interés por parte de los autores.

Fecha de recepción: 22 de diciembre de 2017

Fecha de aceptado: 25 de enero de 2018

\section{ORCID}

https://orcid.org/0000-0002-3371-0987

\section{Correspondencia:}

Miguel Calva Maldonado

Benemérita Universidad Autónoma de Puebla

Hospital Universitario de Puebla.

25 poniente 1301 Col. Volcanes, Puebla México C.P.72410, Teléfono: (01222) 2295500 ext. 6000

E-mail: drcalva55@gmail.com 
se presenta el caso de una paciente de 58 años programada para la resección de tumoración cutánea temporo-parietal derecha, se utilizó succinilcolina como relajante muscular en donde a pesar de la vida media corta de éste, no se requirió de dosis subsecuentes durante el tiempo de duración de la cirugía; por lo que se corroboro una sensibilidad alterada hacia los relajantes musculares de tipo despolarizantes en este tipo de pacientes.

\section{Abreviaturas}

ASA: Asociación americana de anestesiología

IMC: Índice de masa corporal

PANI: Presión arterial no invasiva

EKG: Electrocardiograma

TOF: Tren de cuatro

NF1: Neurofibromatosis tipo 1

\section{Introducción}

a enfermedad de Von Recklinghausen o neurofibromatosis tipo I forma parte de un grupo de enfermedades de herencia autosómica dominante caracterizada por tendencia a la formación de tumores de tejidos del ectodermo y mesodermo. Es de carácter autosómico dominante, afecta a uno de cada 3.000 - 4.000 nacidos vivos[1],[2].

Se describió por primera vez en 1882 la neurofibromatosis tipo 1, como una de las enfermedades de causa monogénica que con mayor frecuencia aparecen en el ser humano. Su fisiopatología consiste en la alteración del gen NF1, un gen supresor de tumores que se localiza en el cromosoma 17q11, localizado en 1987 y aislado en 1990[3].

Los criterios para el diagnóstico clínico se establecieron desde 1988, a partir de dos o más manchas color café con leche de más de cinco milímetros de diámetro tienen característicamente neurofibromatosis múltiples, cutáneos o plexiformes (tumores benignos formados por células de Schwann y fibroblastos), manchas café con leche (crecimiento análogo de melanocitos) y nódulos de Lisch (hamartomas de iris), así como una elevada incidencia de neurofibrosarcomas malignos y gliomas ópticos, además de anomalías óseas, retraso mental y dificultades para el aprendizaje[3],[4].

Existe una gran cantidad de literatura que advierte las implicaciones anestésicas de pacientes con NF1; éstas incluyen la sensibilidad alterada a la succinilcolina y a relajantes neuromusculares no-despolarizantes[7].

Algunos reportes indican que los pacientes con NF1 deben ser observados de cerca por cualquier signo de anormalidad vascular y peligro de ruptura vascular. Además de las implicaciones de la vía aérea difícil, secundaria a la formación de neurofibromas en la lengua y otras estructuras intraorales aledañas. Deformidades

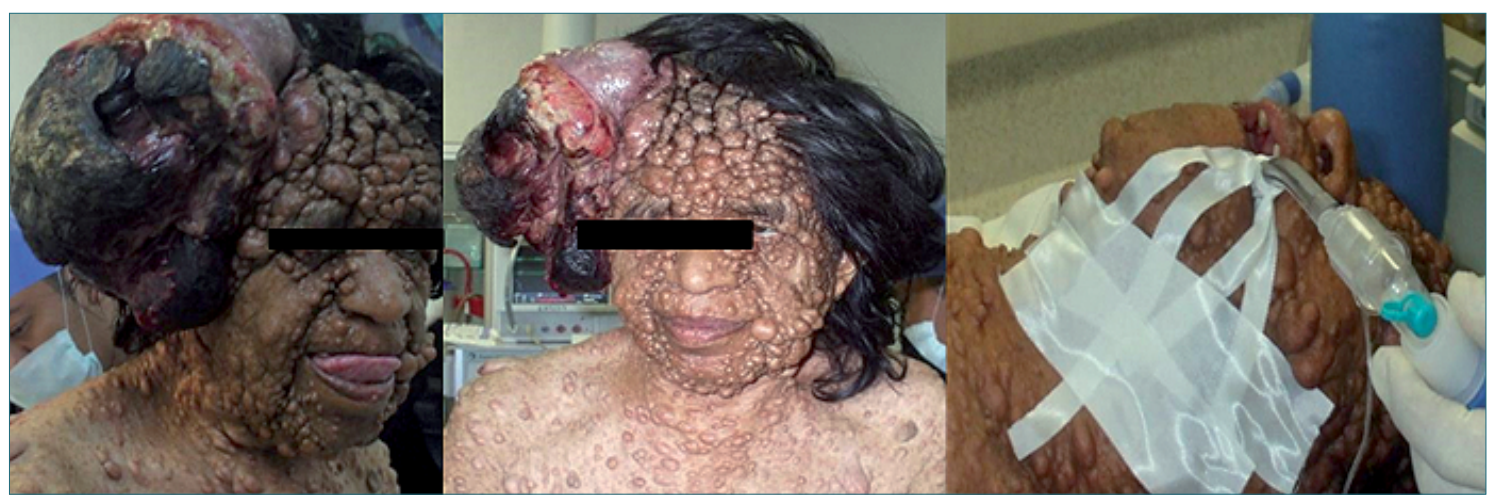

Figura 1. 
de la pared torácica, hipertensión arterial, cardiomiopatía, enfermedad renovascular, coartación de la aorta, tumores carcinoides y feocromocitoma[5],[6].

\section{Caso clínico}

Paciente de sexo femenino de 58 años, con antecedentes de Neurofibromatosis tipo 1, ASA 1. Se programa para resección de tumoración cutánea temporo-parietal derecha de aproximadamente 20 $\mathrm{cm} \times 15 \mathrm{~cm} \times 15 \mathrm{~cm}$ de bordes irregulares, indolora de crecimiento gradual que se acompaña de secreción purulenta fétida.

A la valoración pre anestésica con la siguiente somatometría: Talla: 1,58 m. Peso: $70 \mathrm{~kg}$. IMC: $28 \mathrm{~kg} / \mathrm{m}^{2}$. Temperatura: $36^{\circ} \mathrm{C} \mathrm{FC:} 78 x^{\prime}$ TA: $130 / 80 \mathrm{mmHg}$. FR: $16 x^{\prime}$, cavidad oral con apertura oral de $4 \mathrm{~cm}$, adoncia parcial de arcada superior con piezas dentarias móviles, macroglosia, mallampati III, cuello corto, patil aldreti. $<6 \mathrm{~cm}$, Bell House Doré: II, Distancia Esternomentoniana: $12 \mathrm{~cm}$. Resto del cuerpo con neurofibromas de diferente diámetro indoloras. Bajo monitorización no invasiva (PANI, Pulsioximetria, EKG, TOF), anestesia general balanceada, premedicación con midazolam 2 mg intravenoso. Se realizó inducción intravenosa con: Sufentanil $35 \mathrm{mcg}$, Propofol 140 mg, Succinilcolina 70 $\mathrm{mg}$, realizando intubación orotraqueal bajo laringoscopia directa con hoja curva articulada \#4 tipo McCoy, observando Cormack-Lehane III, colocando cánula orotraquel Sheridan \# 7.5; se realiza mantenimiento Fi02 al 70\%, Sevoflorano a 1 CAM, con parámetro ventilatorio en modo asisto controlado por volumen. Al finalizar la cirugía, la monitorización neuromuscular con TOF resulto de $90 \%$ a los 90 min, no requiriendo dosis subsecuente de relajante muscular.

\section{Discusión}

Se decide realizar el reporte del caso debido a la baja prevalencia de la patología a nivel mundial, como se ha comentado y escasas revisiones sobre el manejo anestésico que existe. La NF-1 significa un reto para el manejo anestésico, por las diversas implicaciones fisiopatológicas y anatómicas existentes, especialmente en el abordaje de la vía aérea por lo que la valoración e identificación de datos de vía aérea difícil, es primordial para la elección de la técnica anestésica y la disponibilidad de aditamentos para su manejo.

En nuestro paciente no existió dificultad para la visualización e instrumentación de la vía aérea, manteniéndose saturación de oxigeno > 95\% y manteniendo estabilidad en sus constantes vitales durante todo el procedimiento quirúrgico. Existen reportes que hacen referencia también que los pacientes con NF-1 deberán ser observados de cerca por cualquier signo de anormalidad vascular y peligro de ruptura vascular, sin embargo en nuestro caso no existió ninguna complicación de este tipo.

Se ha descrito en algunos pacientes sensibilidad alterada a los relajantes neuromusculares despolarizantes, por un mecanismo aún no descrito que produce la prolongación del efecto, en nuestro caso se decidió el uso de relajante muscular succinilcolina; y que a pesar de la vida media corta de éste, no se requirió de dosis subsecuentes durante el tiempo de duración de la cirugía; por lo que consideramos que se corrobora lo mencionado en la literatura descrita, en la cual se reporta una sensibilidad alterada hacia los relajantes musculares de tipo despolarizante. Se logró realizar el procedimiento quirúrgico sin incidentes y se extuba sin complicaciones, egresando la paciente al área de recuperación pos-anestésica.

\section{Referencias}

1. Korf BR. Neurocutaneous syndromes: Neurofibromatosis I, neurofibromatosis 2, and tuberous sclerosis. Curr Opin Neurol 1997; 10:131-136.

2. Crowe FW, Schull WJ, Neel JV. A clinical, Pathological, and Genetic Study of Multiple Neurofibromatosis: Charles $C$. Thomas Springfield, IL (EUA), First edition, 1956

3. Lozada $\mathrm{Y}$, Álvarez-Valiente $\mathrm{H}$, Argüelles M. Estudio clínico de la neurofibromatosis tipo 1. Rev. Neurol 2008; 27(159):792-95.

4. Sisto L. La anestesia en las enfermedades neurológicas. La Habana: Editorial Pueblo y Educación; 2002. p 13-15.

5. Sakai M, Vallejo MC, Shannon $\mathrm{KT}$. A parturient with neurofibromatosis type 2: anesthetic and obstetric considerations for delivery. Inter J Obst Anesth 2005; 14:332-335.

6. Hirsch NP, Murphy A, Radcliffe JJ. Neurofibromatosis: clinical presentations and anaesthe- tic implications. Br J Anaesth 2001;86:555-564.

7. Kamath Sriganesh, DM. Dhritiman Chakrabarti, MD. Tanmay Jadhav, MD. Sudhir Venkataramaiah, MD; Can J Anesth/J Can Anesth (2015) 62:10171018.

8. Developing Cervical Hematoma Following Jaw Thrust Maneuver Triggered Diagnosis of Neurofibromatosis: A Case Report and Brief Literature Review; Anesth Pain Med. 2017 June; 7(3):e41941. 